\title{
Evaluation of Causality Between ADHD and Parkinson's Disease:
}

\section{Mendelian Randomization study}

Gloria Hoi-Yee Li, PhD*, ${ }^{1}$ Grace Mengqin Ge, MSc* ${ }^{1}$ Ching-Lung Cheung, $\mathrm{PhD}^{1,2,3}$, Patrick Ip, FRCPCH(UK) $)^{4}$, David Coghill, $\mathrm{MD}^{5,6}$, Ian Chi-Kei Wong, $\mathrm{PhD}^{1,7}$

*These authors contributed equally.

${ }^{1}$ Department of Pharmacology and Pharmacy, Li Ka Shing Faculty of Medicine, The University of Hong Kong, Hong Kong. ${ }^{2}$ Centre for Genomic Sciences, Li Ka Shing Faculty of Medicine, The University of Hong Kong, Hong Kong. ${ }^{3}$ Department of Medicine, Li Ka Shing Faculty of Medicine, The University of Hong Kong, Hong Kong. ${ }^{4}$ Department of Paediatrics and Adolescents Medicine, Li Ka Shing Faculty of Medicine, The University of Hong Kong, Hong Kong. ${ }^{5}$ Departments of Paediatrics and Psychiatry, University of Melbourne, Melbourne, 3052, Australia. ${ }^{6}$ Murdoch Children's Research Institute, Melbourne, 3052, Australia. ${ }^{7}$ Research Department of Practice and Policy, School of Pharmacy, University College London, London, UK.

\section{Correspondence and reprint requests:}

Ian CK Wong, $\mathrm{PhD}$

Department of Pharmacology and Pharmacy

The University of Hong Kong

Pokfulam, HONG KONG

Email : wongick@hku.hk
Ching-Lung Cheung, $\mathrm{PhD}$

Department of Pharmacology and Pharmacy The University of Hong Kong

Pokfulam, HONG KONG

Email: lung1212@hku.hk

Key words: ADHD, Parkinson's disease, mendelian randomization 


\section{Abstract}

In a retrospective cohort study, patients with attention-deficit hyperactivity disorder (ADHD) and psychostimulant prescription were associated with increased risk of Parkinson's disease (PD). It is unclear whether ADHD per se or psychostimulant prescription is associated with PD. We aim to determine if genetic correlation or/and causal association exists between ADHD and PD using summary statistics obtained from the largest meta-analysis of genome-wide association studies of ADHD (20,183 cases; 35,191 controls) and PD (26,421 cases; 442,271 controls). Genetic correlation was tested between ADHD and PD by linkage disequilibrium score regression. Causal estimate was assessed by inverse-variance weighted (IVW) method as the main mendelian randomization analysis, with sensitivity analyses to detect horizontal pleiotropy. Weak and inverse genetic correlation existed between ADHD and PD ( $\mathrm{r}=-$ $0.100 ; \mathrm{SE}=0.045 ; \mathrm{P}=0.026)$. Univariable IVW analysis with 10 and 77 genetic instruments respectively revealed null association for ADHD with $\mathrm{PD}(\mathrm{OR}=0.930$ per doubling in odds of ADHD; 95\% CI:0.792-1.092) and PD with ADHD (OR=0.986 per doubling in odds of PD; 95\% CI:0.956-1.015). Multivariable IVW analyses adjusted for BMI/smoking also revealed null association of ADHD with PD. Using 58 PD-associated genetic instruments, multivariable IVW analysis with/without adjustment for BMI/smoking suggested a weak and inverse causal association for PD on ADHD, but cautious interpretation is required. This well-powered study did not support causality between ADHD and PD. The observed positive association between ADHD and PD is more likely to be caused by unmeasured confounders. As psychostimulant use is associated with high risk of early-onset PD, future research should focus on this area. 


\section{Introduction}

Being one of the most prevalent neurodevelopmental disorders across the lifespan, attentiondeficit hyperactivity disorder (ADHD) is characterized by inattention, hyperactivity, and increased impulsivity, leading to impairment on an individual's daily functioning across various settings(Bonvicini et al., 2016). The prevalence of ADHD is estimated to be approximately 5\% in school-aged children and 2.5\% in adults(Lopez et al., 2018; Polanczyk et al., 2014; Thomas et al., 2015). Functional neuroimaging finds that ADHD patients have dysfunction of brain regions including the frontal cortex, limbic system, basal ganglia, and reticular activating system, and suggests that ADHD is caused by deficits in dopaminergic and noradrenergic neurotransmission(Curtin et al., 2018; Levy and Psychiatry, 1991; Tripp and Wickens, 2009). Current pharmacological treatment of ADHD includes the psychostimulants, methylphenidate (MPH) and amphetamine derivatives (AMP), both of which regulate dopamine and norepinephrine in the brain by reversibly blocking the dopamine reuptake transporter (DAT)(Attention-Deficit and DISORDER, 2011; Volkow et al., 2005).

Parkinson's disease (PD) is the second most common progressive neurological disorder that affects at least six million people globally(De Lau and Breteler, 2006; Dorsey et al., 2018). The neuropathological feature of PD is the presence of $\alpha$-synuclein-containing Lewy bodies and a pronounced reduction in striatal dopamine, manifesting as severe motor symptoms, including uncontrollable tremor, postural imbalance, slower movement and rigidity(De Lau and Breteler, 2006; Lotharius and Brundin, 2002). It has been observed in a case-control study that ADHD symptoms may precede PD, with no evidence of involvement of psychostimulants(Walitza et al., 2007). However in a recent large retrospective cohort study using medical records between 1996 and 2016 from Utah Population Database (with 31,769 patients with ADHD and 158,790 non-ADHD persons), where patients with ADHD were associated with more than 2-fold 
increased risk of early-onset PD or basal ganglia and cerebellum (Parkinson's like) diseases, there was also 8.6-fold increased risk of developing early-onset PD or Parkinson's like diseases for those ADHD patients who were prescribed psychostimulants(Curtin et al., 2018). Curtin et al considered the excessive risk in patients treated with psychostimulants is attributed to the severity of the disease rather than psychostimulants(Curtin et al., 2018). It is therefore important to evaluate the association between ADHD and PD. Moreover, our previous study has shown significant increase in the use of ADHD medication in children and $4.5 \%$ of children in the North America have been prescribed ADHD medication(Raman et al., 2018), implying a potential public health issue if psychostimulants would increase the risk of developing PD. Currently, it remains unclear whether ADHD has a positive causal effect on PD, or whether the association between the two diseases is due to shared mechanisms. Moreover, PD is influenced by both genetic and environmental factors. It is also possible that the genetic factors of PD also play a causal role in ADHD development, with people susceptible to PD having increased risk of developing ADHD, which is usually manifested at a younger age. With exposure to environmental factors, such as pesticides, disease symptoms of PD may subsequently appear in individuals at a later stage of life.

Mendelian randomization (MR) is considered a powerful approach which makes use of genetic variation as natural and random experiment to evaluate the causal association between two traits or diseases, when such relationships cannot be directly evaluated using clinical trial(Emdin et al., 2017). Its principles were described clinically elsewhere(Emdin et al., 2017). In brief, genetic variants that influence the susceptibility to a disease (like ADHD as exposure) identified from genome-wide association studies (GWAS) could serve as instruments to determine the association of lifelong risk of another disease (like PD as outcome). The naturally assigned genetic variants utilized in MR analyses were fixed at conception, making MR 
approach less likely to be influenced by unmeasured confounding and reverse causation when compared to observational studies, relying on several assumptions(Davies et al., 2018). These assumptions for MR and our study design are illustrated in Figure 1.

Based on the findings of the aforementioned large-scale retrospective cohort study, we hypothesized that ADHD might have a positive causal role on PD or vice-versa, which may contribute to the increased risk of PD in ADHD patients, especially for those who were prescribed psychostimulants. Therefore, the aim of this MR study is to examine the causal relationship between ADHD and PD, and study the effect of potential mediators in such relationship.

\section{Experimental Procedures}

\section{Data sources}

Details of the data sources are listed out in Table 1. Summary statistics of genetic instruments were extracted from the largest publicly available GWAS / GWAS meta-analysis of the exposure, outcome and potential mediators. The risk factor and outcome were ADHD $(20,183$ cases and 35,191 controls)(Demontis et al., 2019) and PD (around 1.4 million subjects in total; the minimum sample size at which the genetic instruments were tested had 26,421 cases and 442,271 controls)(Nalls et al., 2019). In previous studies, smoking and BMI were found to be genetically correlated with both ADHD(Demontis et al., 2019) and PD(Nalls et al., 2019) although the direction was opposite. Higher BMI was reported to be causally associated with increased risk of ADHD(Martins-Silva et al., 2019) but decreased risk of PD(Noyce et al., 2017). It is possible that ADHD and PD might be linked up by smoking or BMI. Datasets of potential mediators under investigation included a GWAS meta-analysis of body mass index (BMI) $(\mathrm{N}=681,275)(Y e n g o$ et al., 2018), and a meta-analysis of 35 GWAS of smoking status 
$(\mathrm{N}=1,232,091$; ever regular versus never regular)(Liu et al., 2019). Ethical approval has been obtained from respective GWAS as stated in the publications.

\section{Estimation of genetic correlation}

Linkage disequilibrium (LD) score regression(Bulik-Sullivan et al., 2015a; Bulik-Sullivan et al., 2015b) was employed to estimate the genetic correlation between ADHD and PD based on the summary statistics of GWAS data(Demontis et al., 2019; Nalls et al., 2019) instead of individual-level genotypes. This method has the advantages of not being biased by sample overlap between GWAS datasets and not requiring multiple phenotype measurements to be conducted on the same individual in estimating genetic correlation between complex traits(Bulik-Sullivan et al., 2015a). We used pre-computed LD scores suitable for Europeanancestry samples and Python command line tool (http://github.com/bulik/ldsc).

\section{Selection of genetic instruments for MR analyses}

Independent genetic variants significantly associated with the exposure (ADHD or PD) were identified by the respective GWAS(Demontis et al., 2019; Nalls et al., 2019). In brief, Demontis et al first performed LD clumping, which started with the variant with the strongest association with ADHD (as the index variant). Other variants $500 \mathrm{~Kb}$ away and uncorrelated $\left(\mathrm{r}^{2}<0.2\right)$ from the existing index variant were assigned to a new locus/clump, until all ADHDassociated genome-wide significant $\left(\mathrm{p}<5 \times 10^{-8}\right)$ variants were assigned to one of the risk loci/clumps. Within each locus/clump, independent index variants were subsequently identified $\left(\mathrm{r}^{2}<0.1\right)$ (Demontis et al., 2019). Meanwhile, independent PD-associated genetic variants $\left(\mathrm{p}<5 \times 10^{-8}\right)$ were identified by Nalls et al in conditional-joint analysis corrected for LD between single nucleotide polymorphisms (SNPs)(Yang et al., 2012), with a window of $>1$ megabase indicating that SNPs are in complete LD(Nalls et al., 2019). The genome-wide 
independent genetic variants uncovered by respective GWAS were initially selected as the genetic instruments in MR analyses. If the summary statistics of a genetic instrument were unavailable in the GWAS of outcome or mediator datasets, the same proxy in high $\operatorname{LD}\left(\mathrm{r}^{2} \geq 0.8\right.$ with reference to the European samples in the 1000 Genome project) and present in all the exposure, outcome and mediator datasets was selected as the genetic instrument. If no proxies could be identified, or if the proxy was no longer significantly associated with the exposure $(\mathrm{P}>0.05)$, the genetic instrument/proxy was excluded from MR analysis. This formed the first part of the analysis. In addition, a large portion of the genome is likely pleiotropic. Whether the genetic instruments/proxies were associated with potential confounders of ADHD/PD (such as education attainment(Arnold et al., 2020; Kotagal et al., 2015), schizophrenia(Gudmundsson et al., 2019; Mok et al., 2016), waist circumference(Chen et al., 2004; Fuemmeler et al., 2011), serum lipid levels(Ugur et al., 2018; Xicoy et al., 2019), neuroticism(Knouse et al., 2013; Sieurin et al., 2016), Crohn's disease(Hegvik et al., 2018; Hui et al., 2018), and inflammatory bowel disease(Hegvik et al., 2018; Villumsen et al., 2019)) were checked at PhenoScanner, a database of publicly available genotype-phenotype association results from GWAS(Staley et al., 2016). Notably, genetic instruments associated with BMI and smoking status were kept in the analyses as they were targeted to be examined in the current study by multivariable MR analyses to determine if BMI and smoking status are potential mediators linking up the two diseases. For genetic variants associated with other potential mediators / confounders as identified by large-scale GWAS conducted by relevant representative consortiums, they were considered as pleiotropic genetic instruments and excluded from this second part of analysis. This approach aims to avoid using instruments that might contribute large effects on multiple traits at the same time. As a sensitivity analysis, more stringent selection criterion were adopted to select the genetic instruments for MR analyses, using the clump command in PLINK 1.9(Chang et al., 2015) with a window $=10 \mathrm{Mb}$ and $\mathrm{r}^{2}$ 
cutoff $=0.001$, which was in line with the MR-Base application(Hemani et al., 2018). In inferring causality of ADHD on PD, 12 independent SNPs significantly associated with ADHD were initially employed as genetic instruments in dissecting the causal effects of ADHD on PD. Similarly, in evaluating the causality of PD on ADHD, 90 independent SNPs significantly associated with PD were initially selected as genetic instruments. Notably, for the PD dataset, the online immediately accessible version of summary statistics does not include the genetic data from 23andMe, Web-Based Study of Parkinson's Disease and the meta-analysis conducted by Nalls et al 2014(Nalls et al., 2014), with a sample size of around 470,000 when compared to the full dataset of around 1.4 million individuals. The association between a genetic proxy and PD might be weak in the online diminished dataset due to the insufficient sample size and reduced power. The summary statistics of the selected genetic instruments for each MR analysis are included in Supplemental Tables 1-6. Pleiotropic associations of the excluded genetic instruments were listed in Supplemental Table 7.

\section{Power calculation}

Proportion of variance in the exposure explained by the genetic instruments was derived from the Mangrove package in R(Jostins et al., 2013). An online web tool, mRnd (http://cnsgenomics.com/shiny/mRnd/)(Brion et al., 2013), was employed to perform power calculation.

\section{MR analyses}

All the genetic instruments were oriented such that the effect alleles were positively associated with the exposure. The effect alleles were matched across the summary data of the exposure, mediator and outcome dataset. Univariable inverse-variance weighted (IVW)(Burgess et al., 2013) method with random-effects model was used for main MR analysis to assess the total 
effect of the exposure on the outcome(Burgess et al., 2017), with heterogeneity between the causal estimates of the genetic instruments tested. Although IVW is the conventional method of MR analysis, the major drawback is that it assumes all instrumental variables are valid. If IVW analysis revealed a significant causal association, weighted median method, MR-Egger and MR-PRESSO's main analysis were used as sensitivity analyses. Weighted median method provides consistent estimates even when up to $50 \%$ of the information comes from invalid instrumental variables(Bowden et al., 2016). Under the assumption that the magnitude of the pleiotropic effects are independent of the SNP-risk factor associations across all variants, also known as the INstrument Strength Independent of Direct Effect (InSIDE) assumption, the intercept of MR-Egger regression represents the average pleiotropic effects across all SNPs(Bowden et al., 2015). With this InSIDE assumption, MR-Egger regression detects and corrects for bias arising from unbalanced pleiotropy in MR studies(Bowden et al., 2015). MREgger intercept test(Bowden et al., 2015) and MR pleiotropy residual sum and outlier (MRPRESSO)(Verbanck et al., 2018) were employed to test for the presence of directional pleiotropy. MR-PRESSO comprises of three components: (i) global test has adequate power to evaluate the overall horizontal pleiotropy among all instruments even if pleiotropy just occurs in less than half of the instruments; (ii) outlier test provides the causal estimate upon removal of pleiotropic genetic instruments; and (iii) distortion test determines if there is significant difference in the causal estimate before and after the removal of pleiotropic genetic instruments. Due to the use of a large number of instruments, heterogeneity between their causal estimates was not evitable and it was regarded as a chance to examine if multiple distinct causal mechanisms existed between the exposure and outcome using the contamination mixture method(Burgess et al., 2020). Multivariable IVW analysis was also performed to dissect the mechanisms in the causal pathway from the exposure to the outcome(Burgess et al., 2017; Burgess and Thompson, 2015). The direct causal effect of the exposure on the outcome was 
evaluated by multivariable IVW by adjusting for the beta estimates of potential mediators, including BMI and smoking status. If multivariable IVW analyses revealed causality, multivariable MR-Egger method was also applied as a sensitivity analysis. Multivariable MREgger intercept test was employed to detect for pleiotropies(Rees et al., 2017). MR-PRESSO analysis was performed using "MRPRESSO" package in R(Verbanck et al., 2018) while univariable IVW, weighted median, MR-Egger and contamination mixture analyses were conducted with 'MendelianRandomization' package in R(Yavorska and Burgess, 2017). Multivariable IVW and MR-Egger analyses were also conducted using the 'MendelianRandomization' package(Yavorska and Burgess, 2017).

As the exposures in all the MR analyses are binary variables, the causal estimates were initially equivalent to the change in the outcome per unit change in the exposure in log odds scale [=exponential 1, i.e. 2.72-fold change in the odds of the exposure]. For the sake of interpretation, the causal estimates were converted by multiplying $0.693(=\ln 2)$ and then exponentiating to represent change in outcome per 2-fold change in the prevalence of the exposure(Burgess and Labrecque, 2018).

\section{Results}

Genetic correlation between ADHD and PD

Genetic correlation between ADHD and PD was evaluated by LD score regression. Weak and inverse genetic correlation was observed between ADHD and PD $(r=-0.100 ; S E=0.045 ; z=-$ $2.220 ; \mathrm{P}=0.026)$.

Two-sample MR of ADHD with PD 
Out of the 12 independent ADHD-associated SNPs, eight SNPs were available in the PD dataset and proxies in high LD with the unmatched SNPs were identified for the remaining four SNPs (For $\mathrm{r}^{2}$ of the four proxies, median: 0.881; range: 0.812-0.975; Supplemental Table 1). Null causal association was observed in univariable ( $\mathrm{OR}=0.900$ per doubling in odds of ADHD; 95\% CI: 0.785-1.032; $\mathrm{P}=0.131$; Table 2), with a significant MR-PRESSO global test $(\mathrm{P}=0.048)$. For multivariable IVW analysis, the 12 ADHD-associated independent SNPs were matched across the datasets of mediators (including smoking and BMI) and outcome (PD). Five of the SNPs could be matched while proxies were identified for the remaining seven unmatched SNPs (For $\mathrm{r}^{2}$ of the seven proxies, median: 0.975; range: 0.812-1; Supplemental Table 2). IVW analysis before and after adjustment for potential mediators resulted in a null causal association, with some evidence of pleiotropy (Before adjustment for mediators: MREgger intercept test $\mathrm{P}=0.023$; MR-PRESSO global test $\mathrm{P}=0.028$; Upon adjustment for $\mathrm{BMI}$ : multivariable Egger intercept test $\mathrm{P}=0.006$; Table 2).

The MR analyses were repeated with exclusion of pleiotropic genetic instruments, as the pleiotropy may violate the MR assumption and affect the validity of the analyses. Two pleiotropic genetic instruments were excluded from MR analyses and a total of 10 genetic instruments were utilized in univariable MR analysis (Supplemental Table 1). Univariable IVW analysis revealed null causal association of $A D H D$ with $P D(O R=0.930$ per doubling in odds of ADHD; 95\% CI: 0.792-1.092; P=0.374; Table 3; Supplemental Figure 1a). Both MREgger intercept test $(\mathrm{P}=0.488)$ and MR-PRESSO global test $(\mathrm{P}=0.071)$ suggested the absence of horizontal pleiotropy (Table 3). For multivariable analysis with instruments matching across the mediator and outcome datasets, three of the proxies were pleiotropic so they were excluded from this part of analyses, and a total of nine genetic instruments were used (Supplemental Table 2). Null causal association between ADHD and PD was observed in univariable (Table 
3; Supplemental Figure 1b) and multivariable IVW analysis before and after adjustment for BMI or smoking status. Consistently, the multivariable Egger intercept tests were insignificant (Adjusted for BMI: $\mathrm{P}=0.451$; Adjusted for smoking status: $\mathrm{P}=0.624$ ), implying no pleiotropy was present (Table 43).

Using contamination mixture method, unimodal distribution of causal estimates was observed in all the above univariable analyses, implying the absence of multiple mechanisms involved. In examining the causal effects of ADHD on PD, majority of the genetic instruments were located on different chromosomes. The only two instruments located on the same chromosome were separated by more than $50 \mathrm{Mb}$ with $\mathrm{r}^{2}<0.001$. Even with more stringent criterion of selecting genetic instruments adopted by MR-Base, the same set of genetic instruments was derived. No supplementary sensitivity analysis was performed.

Two-sample MR of PD with ADHD

Out of the 90 PD-associated SNPs, 85 SNPs could be matched with the ADHD dataset and proxy could be identified for one of the remaining unmatched SNPs $\left(r^{2}=0.921\right.$; Supplemental Table 3). In univariable IVW analysis, null causal association was observed $(\mathrm{OR}=0.980 ; 95 \%$ CI: 0.955-1.005; $\mathrm{P}=0.114$; Table 4). Horizontal pleiotropy was unlikely (MR-Egger intercept: $\mathrm{P}=0.841$; MR-PRESSO global test: $\mathrm{P}=0.127$ ). For multivariable analysis, we matched the 90 PD-associated SNPs across the datasets of BMI, smoking status and ADHD. 34 of the SNPs were available across the datasets and proxies were identified for 38 of the remaining unmatched SNPs (For $\mathrm{r}^{2}$ of the 38 proxies, median: 1; range: 0.824-1; Supplemental Table 4). Two proxies were no longer associated with PD and were excluded from analysis, leaving 70 genetic instruments. Before adjustment for potential mediators, doubling in odds of PD was 
associated with reduced risk of ADHD (IVW: $\mathrm{OR}=0.967 ; 95 \% \mathrm{CI}: 0.937-0.997 ; \mathrm{P}=0.032$; Table 4). Similar estimate was obtained using weighted-median and MR-PRESSO analysis. Evidence of horizontal pleiotropy was absent (MR-Egger intercept: $\mathrm{P}=0.731$; MR-PRESSO global test: $\mathrm{P}=0.155)$. Multivariable IVW adjusted for the beta estimates of $\mathrm{BMI}$ and smoking status respectively demonstrated that doubling in odds of PD was associated with lower risk of ADHD (Adjusted for BMI: OR=0.967; 95\% CI: 0.938-0.999; $\mathrm{P}=0.039$; Adjusted for smoking status: $\mathrm{OR}=0.963 ; 95 \% \mathrm{CI}$ : 0.934-0.992; $\mathrm{P}=0.011$; Table 4). Meanwhile, multivariable Egger test suggested null association after adjusting for the potential mediators, with insignificant intercept tests $(\mathrm{P}>0.05)$, indicating the absence of pleiotropy.

After excluding nine pleiotropic genetic instruments, a total of 77 genetic instruments were utilized in univariable MR analysis (Supplemental Table 3). Univariable IVW analysis revealed null causal association $(\mathrm{OR}=0.986$ per doubling in odds of $\mathrm{PD}$; 95\% CI: 0.956-1.015; $\mathrm{P}=0.341$; Table 5; Supplemental Figure 2a). Both MR-Egger intercept test $(\mathrm{P}=0.234)$ and $\mathrm{MR}$ PRESSO global test $(\mathrm{P}=0.063)$ did not detect any horizontal pleiotropy (Table 5). For multivariable analysis, 12 genetic instruments were pleiotropic. They were excluded from the second part of analysis, making up a total of 58 genetic instruments (Supplemental Table 4). Before adjustment for potential mediators, IVW analysis demonstrated that doubling in odds of $\mathrm{PD}$ reduced the risk of $\mathrm{ADHD}$ by $4.3 \%(\mathrm{OR}=0.957 ; 95 \% \mathrm{CI}: 0.925-0.991 ; \mathrm{P}=0.013)$ (Table 5; Supplemental Figure 2b). Sensitivity analysis of weighted median method yielded similar estimates $(\mathrm{OR}=0.930 ; 95 \% \mathrm{CI}: 0.883-0.979 ; \mathrm{P}=0.006)$. Null association was observed in MREgger test $(\mathrm{OR}=0.943 ; 95 \% \mathrm{CI}: 0.866-1.026 ; \mathrm{P}=0.173)$. MR-Egger intercept test $(\mathrm{P}=0.696)$ and MR-PRESSO global test $(\mathrm{P}=0.145)$ did not detect any horizontal pleiotropy. Similar causal association for PD on ADHD was observed after adjustment for BMI (OR=0.958; 95\% CI: 0.926-0.992; $\mathrm{P}=0.015)$ and smoking status $(\mathrm{OR}=0.948 ; 95 \% \mathrm{CI}: 0.918-0.979 ; \mathrm{P}=0.001)$ (Table 
5). Whereas, null causal association was revealed by multivariable Egger analyses after adjustment for both potential mediators. The multivariable Egger intercept tests were insignificant (Adjusted for $\mathrm{BMI}$ : $\mathrm{P}=0.545$; Adjusted for smoking status: $\mathrm{P}=0.718$ ).

As a sensitivity analysis, stricter criterion with clumping window of $10 \mathrm{Mb}$ and $\mathrm{r}^{2}$ of 0.001 were adopted in selecting genetic instruments. Twenty-four independent SNPs were identified to be associated with PD by clumping. In univariable analysis, twenty-two of the SNPs were matched with the dataset of ADHD while proxy could be identified for one of the unmatched SNPs $\left(r^{2}=0.902\right.$; Supplemental Table 5). Similar null causal association was observed, with some evidence of pleiotropy (MR-PRESSO global test: $\mathrm{P}=0.012$; Supplemental Table 8). For multivariable analysis, eight SNPs were matched across the datasets of mediators and outcome while proxies were identified for the 12 remaining unmatched SNPs (For $\mathrm{r}^{2}$ of the 12 proxies, median: 1; range: 0.860-1; Supplemental Table 6). IVW analyses demonstrated null association between PD and ADHD before and after adjustment for potential mediators, with multivariable Egger intercept tests suggesting the presence of horizontal pleiotropy $(\mathrm{P}<0.05$; Supplemental Table 8).

This sensitivity analysis was repeated after removing the potential pleiotropic instruments. In univariable analysis, two pleiotropic instruments were excluded from the second part of analysis, leaving 21 genetic instruments (Supplemental Table 5). In univariable analysis, similar null causal association was observed with no evidence of pleiotropy (Supplemental Table 9). Two pleiotropic instruments were also removed from the multivariable analysis. Both before and after adjustment for potential mediators, there was null association between PD and ADHD, and pleiotropy was unlikely (Supplemental Table 9). 
In all the univariable MR analyses evaluating the causal effects of PD on ADHD, unimodal distribution of causal estimates was observed using contamination mixture method, implying the absence of multiple causal mechanisms.

\section{Power}

Due to the possible violation of MR assumption, MR analyses with exclusion of pleiotropic genetic instruments were considered as the primary analysis. The statistical power and strength of genetic instruments for the primary MR analysis were presented in Table 6. Since the summary statistics of each genetic instrument might be obtained from a subset of GWAS sample, the minimum sample size was adopted to compute the minimum statistical power of our analyses. Assuming the genuine underlying association had an OR of 1.2 of the outcome per doubling in odds of exposure, the primary analyses had $>90 \%$ power to detect such association. In multivariable MR analysis evaluating the causal effect of ADHD on PD, our study had $\geq 80 \%$ power to detect the causal association if the genuine $\mathrm{OR} \geq 1.167$ or $\mathrm{OR} \leq 0.824$. For the causal effect of PD on ADHD, the study also had $\geq 80 \%$ power to detect the causal association if the $\mathrm{OR} \geq 1.126$ or $\mathrm{OR} \leq 0.883$.

\section{Discussion}

In the two-sample bi-directional MR study, null causal association between ADHD and PD was observed in the univariable analysis, implying the total causal effect between the two disorders was null. Similarly, multivariable MR analyses with adjustment for potential mediators, such as BMI and smoking, did not provide sufficient evidences to demonstrate their roles as mediator between the two disorders. Since the current study is well-powered, the null 
association observed is likely to be genuine. The present study also demonstrated that weak and inverse genetic correlation existed between ADHD and PD.

Both ADHD and PD are heritable, with heritabilites estimated to be 0.22 for both diseases in the latest GWAS meta-analysis using LD score regression method(Demontis et al., 2019; Nalls et al., 2019). Large-scale GWAS meta-analyses have identified several susceptibility loci, which can be used to evaluate the relationship between ADHD and PD. Genetic correlation analysis revealed the shared genetic architecture between ADHD and PD. In this study, we observed that ADHD and PD were inversely correlated with each other, indicating that genetic variants leading to increased risk of ADHD are associated with reduced risk of PD; however, the association is weak. To enhance the understanding on the pathogenesis of the two genetically correlated diseases, future studies, such as gene-based and enriched pathway analyses, are warranted to examine the genetic overlap and difference between ADHD and PD. The present finding of weak genetic correlation was inconsistent with the null genetic correlation observed(Demontis et al., 2019; Nalls et al., 2019) with the use of smaller datasets of $\mathrm{ADHD}$ and $\mathrm{PD}$ derived from 9 population-based pediatric ADHD cohorts comprising 17,666 children(Middeldorp et al., 2016) and a Caucasian PD cohort with 5,691 participants(Simon-Sanchez et al., 2009) respectively. In particular, the previous GWAS metaanalysis of ADHD could not identify any genome-wide significant signals for the disorder(Middeldorp et al., 2016). Their null genetic correlation may be attributed by the limited power of the GWAS.

We performed MR analyses using different sets of genetic instruments based on different selection criterion. In the first part of the analysis using all exposure-associated SNPs as the genetic instruments, some evidence of pleiotropy was shown by MR-Egger intercept and MR- 
PRESSO global tests. Potential pleiotropic instruments were therefore identified by PhenoScanner (Supplemental Table 7) and excluded from the second part of the analysis, where horizontal pleiotropy was unlikely. Since the presence of pleiotropy would violate MR assumption and might invalidate the MR findings, we considered the analyses with exclusion of pleiotropic instruments as the primary analyses of our study. Additional sensitivity analyses were also performed utilizing stricter selection criterion adopted by MR-Base. In the primary MR analysis, we found no causal association between ADHD and PD, which is indeed consistent with a previous small-scale genetic association study evaluating the relationship of nine ADHD risk variants with $\mathrm{PD}($ Geissler et al., 2017). However, the null association observed in that European study could be due to small sample size (5,333 PD cases and 12,019 controls) and hence limited power. Moreover, they did not evaluate the causal association using MR approach. In the current study, estimates from 26,421 PD cases and 442,271 controls (minimum sample size at which the genetic instruments were tested) were adopted in the MR analysis. Still, no significant causal association was observed. The current well-powered study provides an evidence that ADHD does not casually increase risk of PD development.

BMI and cigarette smoking were individually associated with ADHD and PD. While the pooled prevalence of obesity (defined by BMI) was greatly increased by $70 \%$ and $40 \%$ respectively in adults and children with ADHD, a two-sample MR study demonstrated that higher BMI might reduce the risk of $\mathrm{PD}$ (Noyce et al., 2017). Not only the prevalence of smoking among individuals with ADHD was significantly higher than that of the general population, but significant association was also observed between the number of ADHD symptoms and lifetime risk of regular smoking(McClernon and Kollins, 2008). Meanwhile, tobacco components, such as nicotine, were speculated to be neuroprotective and contribute to the reduced PD risk among tobacco smokers in dose-dependent manner(Ascherio and 
Schwarzschild, 2016). BMI and smoking had a heritable component, with heritabilities of 0.192 and 0.054 respectively derived by LD score regression(Demontis et al., 2019). Previous studies also revealed that BMI and smoking were genetically correlated with ADHD and PD although the direction was opposite in the two disorders. BMI was found to have positive and inverse genetic correlation with $\mathrm{ADHD}\left(\mathrm{r}=0.258 ; \mathrm{SE}=0.032 ; \mathrm{z}=7.96 ; \mathrm{P}=1.68 \times 10^{-15}\right)($ Demontis et al., 2019) and $\mathrm{PD}(\mathrm{r}=-0.062 ; \mathrm{SE}=0.024 ; \mathrm{z}=-2.556 ; \mathrm{P}=0.011)$ respectively(Nalls et al., 2019). Whereas, number of cigarettes smoked per day had positive and modest genetic correlation with ADHD ( $\left.\mathrm{r}=0.451 ; \mathrm{SE}=0.103 ; \mathrm{z}=4.40 ; \mathrm{P}=1.07 \times 10^{-5}\right)($ Demontis et al., 2019), but inverse genetic correlation with $\mathrm{PD}(\mathrm{r}=-0.192 ; \mathrm{SE}=0.071 ; \mathrm{z}=-2.713 ; \mathrm{P}=0.007)($ Nalls et al., 2019). Thus, it is possible that the null association observed in univariable IVW is due to the opposite effect of these potential mediators in the two disorders. Notably, there was weak though significant causal association for $\mathrm{PD}$ on $\mathrm{ADHD}$ when 58 genetic instruments were used in the multivariable IVW analysis (Table 5). However, sensitivity analysis of multivariable MREgger method and using strictly selected genetic instruments did not yield significant causal association. Such inconsistent findings would require cautious interpretations and further studies are required to confirm if such negative causality is genuine. Even if such causal association is genuine, the direction of association was opposite to our hypothesis, i.e. ADHD increases risk of PD or vice-versa. This observation further strengthens the possibility that the observed increased risk of PD among ADHD patients is contributed by other factors (such as psychostimulants and other shared risk factors), instead of the causal relationship between these two diseases.

The current study has several important clinical implications. Our findings did not support causal association exists between $\mathrm{ADHD}$ and $\mathrm{PD}$. In addition to the findings from the retrospective study using medical records from the Utah Population Database(Curtin et al., 
2018), medications of ADHD instead of the disorder itself, may be the contributing factor for increased risk of developing PD among ADHD patients. Given their robust clinical efficacy(Cortese et al., 2018), psychostimulants including methylphenidate (MPH) and amphetamines (AMP) have been the mainstay of treatment for ADHD for the last two decades and widely used in many countries(Raman et al., 2018). Early studies have shown that high doses of AMP might damage dopaminergic pathways(Advokat, 2007; Arnsten, 2006). MPH might have dopamine neurotoxic potential(Wang et al., 2013; Yuan et al., 1997), and such damage may be irreversible. Yet, only limited number of studies have examined the pharmacological risk of psychostimulants and further investigations will be required. A previous empirical assessment reported the exposure to a high dose of MPH or AMP lead to persistent basal ganglia dopaminergic deficits, which suggested patients who abuse MPH or AMP are more likely to develop PD(Gerlach et al., 2003). This is in line with the Utah cohort study that ADHD patients who had been prescribed stimulant medications had an even greater increased risk of developing PD by six to eight fold(Curtin et al., 2018). While the current study eliminated the possibility that the increased PD risk was caused by ADHD, a safety concern arises: ADHD medications may be associated with increase in risk of PD. Nevertheless, other shared risk factors, including brain injury, environmental factors such as exposure to toxic chemicals and metals, can also put an individual at greater risk for being diagnosed with both ADHD and PD(Banerjee et al., 2007; Gorell et al., 1997; Hubble et al., 1993; Priyadarshi et al., 2001). Given that the causal relationship between ADHD per se and PD is unlikely to be present, as demonstrated in the current study, whether psychostimulants that are commonly used to treat ADHD would lead to increased risk of PD warrants further investigation. The European ADHD Guidelines Group has recommended that continued monitoring of potential neurological adverse effects in older patients is needed(Wong et al., 2019). Further research should focus on this area. 
The key strength of our study is the adoption of two-sample MR study to evaluate the causal association between two diseases, which is infeasible to be assessed by clinical trials. We utilized summary statistics derived from the largest GWAS meta-analysis of ADHD and PD to-date. These well-powered GWAS meta-analyses provided strong genetic instruments for the current MR study. The relatively high F-statistics suggested that our study unlikely suffers from weak instrument bias. We have over $90 \%$ power to detect association with an OR of 1.2 per doubling in odds of exposure in this study, implying that the null association observed is likely to be genuine. In addition, sample overlap between the exposure and outcome datasets was unlikely, avoiding bias that would arise in the direction of confounded association if overlapping sample increases(Burgess et al., 2016). Nevertheless, there are limitations. First, presence of horizontal pleiotropy may be inevitable that the genetic instruments may have an indirect effect on the outcome via a pathway that does not involve the risk factor of interest. However, we have used two different methods to test for the presence of horizontal pleiotropy (MR-Egger intercept and MR-PRESSO global tests) and found no evidence of pleiotropy upon exclusion of potentially pleiotropic instruments, though horizontal pleiotropy cannot be ruled out unequivocally. Second, the original instruments significantly associated with the exposure may not be available across the datasets of mediators and outcome in multivariable MR analyses. Proxies in high LD with the original instruments present in all the datasets were adopted in the multivariable analysis. However, these proxies were identified based on the LD pattern of Europeans in 1000 Genome Project, instead of the LD pattern in the respective GWAS cohorts. Although most of the genetic proxies had effect size and standard error comparable to that of original instruments regarding their association with the exposure, a few proxies had relatively different summary statistics. Studies with individual-level genotyping data may be warranted to identify reliable proxies of high LD in the GWAS cohort. Third, like 
many other epidemiological studies, there are possible unmeasured and residual confounding factors. Fourth, while the GWAS of PD, BMI and smoking status were conducted in European participants, approximately $95 \%$ of the individuals in the ADHD dataset were of European ancestry. This may lead to violation of MR assumption as association between a genetic variant and the disease outcome may be confounded by population substructure(Davies et al., 2018). Nonetheless, the results of GWAS meta-analysis of ADHD in Europeans were similar to that in ethnically mixed population(Demontis et al., 2019), suggesting that the 5\% population admixture may not confound the genetic association. Fifth, the GWAS were conducted in participants of mainly European ancestry, generalization of the findings to other ethnic groups need to be cautious.

\section{Conclusions}

Our study findings suggest no causal association exists between ADHD and PD. Although previous studies showed BMI and smoking status had significant yet opposite genetic correlation with ADHD and PD respectively, they are unlikely the mediators of the two diseases. The positive association between ADHD and increased PD risk observed in a recent retrospective study is more likely to be caused by other unmeasured confounders that warrant further investigation. As previous study showed psychostimulant use is associated with remarkably high risk of subsequent early-onset PD and Parkinson's like diseases, future research should focus on this area. 


\section{Author Disclosures}

This study does not receive any funding. None of the authors have any financial conflicts of interest to disclose.

CLC, ICW, PI and DC contributed to the conception of the work. CLC and ICW designed the study. CLC and GHL performed the data analysis. GHL, GMG, CLC and ICW had full access to all the data in the study and take responsibility for the integrity of the data and the accuracy of the data analysis. GHL, GMG and CLC wrote the first draft of the manuscript. GHL, GMG, CLC, PI, DC and ICW interpreted, critically evaluated, and improved the study design and manuscript, and shared the responsibility for the final manuscript and the decision to submit. 
Table 1. Data sources of the diseases / traits included in genetic correlation and/or MR analyses

\begin{tabular}{|c|c|c|c|c|c|}
\hline & Disease or trait & $\begin{array}{l}\text { Exposure / outcome / } \\
\text { mediating factor in } \\
\text { MR analyses? } \\
\end{array}$ & Description of data source & Ancestry & Sample size \\
\hline 1 & $\begin{array}{c}\text { Attention- } \\
\text { Deficit/Hyperactivity } \\
\text { Disorder (ADHD) }\end{array}$ & Exposure / Outcome & $\begin{array}{l}\text { A GWAS meta-analysis from } 12 \\
\text { cohorts that identified the first genome- } \\
\text { wide significant ADHD loci(Demontis } \\
\text { et al., 2019). }\end{array}$ & $\begin{array}{l}\text { Majority } \\
\text { European } \\
\text { (approximately } \\
95 \% \text { ) }\end{array}$ & $\begin{array}{l}\text { Cases: } 20,183 \text {; } \\
\text { Controls: } 35,191\end{array}$ \\
\hline 2 & $\begin{array}{c}\text { Parkinson's Disease } \\
\text { (PD) }\end{array}$ & Exposure / Outcome & $\begin{array}{l}\text { The largest GWAS meta-analysis of PD } \\
\text { to-date, including three published } \\
\text { studies and } 13 \text { new case-control sample } \\
\text { series(Nalls et al., 2019). }\end{array}$ & European & $\begin{array}{l}\text { (a) Cases: 26,421 } \\
\text { Controls: } 442,271\end{array}$ \\
\hline 3 & $\begin{array}{l}\text { Body mass index } \\
\text { (BMI) }\end{array}$ & Mediating factor & $\begin{array}{l}\text { A meta-analysis of UK Biobank data } \\
\text { with a previous GWAS of the Genetic } \\
\text { Investigation of ANthropometric Traits } \\
\text { (GIANT) consortium(Yengo et al., } \\
\text { 2018). }\end{array}$ & European & 681,275 \\
\hline 4 & $\begin{array}{l}\text { Smoking status (ever } \\
\text { regular vs never } \\
\text { regular) }\end{array}$ & Mediating factor & $\begin{array}{l}\text { A meta-analysis of } 35 \text { GWAS of } \\
\text { multiple stages of tobacco use and } \\
\text { alcohol use(Liu et al., 2019). }\end{array}$ & European & $1,232,091$ \\
\hline
\end{tabular}

@Minimum sample size at which the genetic instruments were tested 
Table 2. Result of Mendelian Randomization analysis in evaluating the causal association between ADHD and PD without excluding pleiotropic genetic instruments.

Method

Odds Ratio

P-value

(95\% Confidence Interval)

\section{Univariable MR analyses with the use of 12 genetic instruments}

(8 independent SNPs identified from the GWAS of ADHD + 4 proxies)

IVW

$0.900(0.785-1.032)$

0.131

Heterogeneity test statistics

$\mathrm{N} / \mathrm{A}$

0.047

MR-Egger (intercept)

N/A

0.524

MR-PRESSO Global Test

N/A

0.048

\begin{tabular}{|c|c|c|}
\hline \multicolumn{3}{|c|}{ Multivariable MR analyses with the use of 12 genetic instruments } \\
\hline \multicolumn{3}{|c|}{ (5 independent SNPs identified from the GWAS of ADHD +7 proxies) } \\
\hline \multicolumn{3}{|c|}{ Before adjustment for potential mediators } \\
\hline IVW & $0.994(0.862-1.147)$ & 0.935 \\
\hline Heterogeneity test statistics & N/A & 0.027 \\
\hline MR-Egger (intercept) & N/A & 0.023 \\
\hline MR-PRESSO Global Test & N/A & 0.028 \\
\hline \multicolumn{3}{|c|}{ Multivariable MR analyses adjusted for BMI } \\
\hline Multivariable IVW & $1.010(0.868-1.174)$ & 0.903 \\
\hline Heterogeneity test statistics & N/A & 0.024 \\
\hline Multivariable Egger (intercept) & N/A & 0.006 \\
\hline \multicolumn{3}{|c|}{ Multivariable MR analyses adjusted for smoking status } \\
\hline Multivariable IVW & $0.999(0.888-1.126)$ & 0.993 \\
\hline Heterogeneity test statistics & N/A & 0.191 \\
\hline Multivariable Egger (intercept) & N/A & 0.143 \\
\hline
\end{tabular}


Table 3. Result of Mendelian Randomization analysis in evaluating the causal association between ADHD and PD after excluding pleiotropic genetic instruments.

Method

Odds Ratio

P-value

(95\% Confidence Interval)

Univariable MR analyses with the use of 10 genetic instruments

(7 independent SNPs identified from the GWAS of ADHD + 3 proxies)

IVW

$0.930(0.792-1.092)$

0.374

Heterogeneity test statistics

N/A

0.049

MR-Egger (intercept)

N/A

0.488

MR-PRESSO Global Test

N/A

0.071

Multivariable MR analyses with the use of 9 genetic instruments

(5 independent SNPs identified from the GWAS of ADHD + 4 proxies)

Before adjustment for potential confounders

$\begin{array}{lcr}\text { IVW } & 1.123(0.996-1.267) & 0.059 \\ \text { Heterogeneity test statistics } & \text { N/A } & 0.952 \\ \text { MR-Egger (intercept) } & \text { N/A } & 0.587 \\ \text { MR-PRESSO Global Test } & \text { N/A } & 0.954 \\ \text { Multivariable MR analyses adjusted for BMI } & & \\ \text { Multivariable IVW } & 1.132(0.999-1.282) & 0.052 \\ \text { Heterogeneity test statistics } & \text { N/A } & 0.928 \\ \text { Multivariable Egger (intercept) } & \text { N/A } & 0.451\end{array}$

Multivariable MR analyses adjusted for smoking status

Multivariable IVW

$1.107(0.973-1.261)$

0.122

Heterogeneity test statistics

$\mathrm{N} / \mathrm{A}$

0.937

Multivariable Egger (intercept)

N/A

0.624 
Table 4. Result of Mendelian Randomization analysis in evaluating the causal association between PD and ADHD without excluding pleiotropic genetic instruments.

Method

Odds Ratio

P-value

(95\% Confidence Interval)

\begin{tabular}{lcc}
\hline Univariable MR analyses with the use of 86 genetic instruments \\
\hline (85 independent SNPs identified from the GWAS of PD + 1 proxy) \\
IVW & $0.980(0.955-1.005)$ & \\
Heterogeneity test statistics & N/A & 0.114 \\
MR-Egger (intercept) & $0.999(0.994-1.005)$ & 0.112 \\
MR-PRESSO Global Test & N/A & 0.841 \\
\end{tabular}

\begin{tabular}{|c|c|c|}
\hline \multicolumn{3}{|c|}{ Multivariable MR analyses with the use of 70 genetic instruments } \\
\hline \multicolumn{3}{|c|}{ (34 independent SNPs identified from the GWAS of PD + 36 proxies) } \\
\hline \multicolumn{3}{|c|}{ Before adjustment of potential mediators } \\
\hline IVW & $0.967(0.937-0.997)$ & $\mathbf{0 . 0 3 2}$ \\
\hline Heterogeneity test statistics & N/A & 0.168 \\
\hline Weighted-median & $0.941(0.899-0.985)$ & 0.009 \\
\hline MR-Egger & $0.956(0.882-1.037)$ & 0.277 \\
\hline MR-Egger (intercept) & N/A & 0.775 \\
\hline MR-PRESSO Global Test & N/A & 0.175 \\
\hline MR-PRESSO main analysis & $0.967(0.937-0.997)$ & 0.036 \\
\hline \multicolumn{3}{|c|}{ Multivariable MR analyses adjusted for BMI } \\
\hline Multivariable IVW & $0.967(0.938-0.999)$ & $\mathbf{0 . 0 3 9}$ \\
\hline Heterogeneity test statistics & N/A & 0.151 \\
\hline Multivariable Egger & $0.955(0.880-1.025)$ & 0.263 \\
\hline Multivariable Egger (intercept) & N/A & 0.725 \\
\hline \multicolumn{3}{|c|}{ Multivariable MR analyses adjusted for smoking status } \\
\hline Multivariable IVW & $0.963(0.934-0.992)$ & 0.011 \\
\hline Heterogeneity test statistics & N/A & 0.379 \\
\hline Multivariable Egger & $0.952(0.883-1.028)$ & 0.213 \\
\hline Multivariable Egger (intercept) & N/A & 0.773 \\
\hline
\end{tabular}


Table 5. Result of Mendelian Randomization analysis in evaluating the causal association between PD and ADHD after excluding pleiotropic genetic instruments.

Method

Odds Ratio

P-value

(95\% Confidence Interval)

\section{Univariable MR analyses with the use of 77 genetic instruments}

(76 independent SNPs identified from the GWAS of PD + 1 proxy)

IVW

$0.986(0.956-1.015)$

0.341

Heterogeneity test statistics

N/A

0.049

MR-Egger (intercept)

N/A

0.234

MR-PRESSO Global Test

N/A

0.063

\begin{tabular}{lcc}
\hline \multicolumn{2}{l}{ Multivariable MR analyses with the use of 58 genetic instruments } \\
(31 independent SNPs identified from the GWAS of PD +27 proxies $)$ \\
Before adjustment of potential confounders \\
IVW & $0.957(0.925-0.991)$ & \\
Heterogeneity test statistics & N/A & $\mathbf{0 . 0 1 3}$ \\
Weighted median method & $0.930(0.883-0.979)$ & 0.171 \\
MR-Egger & $0.943(0.866-1.026)$ & $\mathbf{0 . 0 0 6}$ \\
MR-Egger (intercept) & N/A & 0.173 \\
MR-PRESSO main analysis & $0.957(0.925-0.991)$ & 0.696 \\
MR-PRESSO Global Test & N/A & $\mathbf{0 . 0 1 6}$ \\
Multivariable MR analyses adjusted for BMI & & 0.145 \\
Multivariable IVW & $0.958(0.926-0.992)$ & \\
Heterogeneity test statistics & N/A & $\mathbf{0 . 0 1 5}$ \\
Multivariable Egger & $0.935(0.858-1.019)$ & 0.170 \\
Multivariable Egger (intercept) & N/A & 0.127 \\
Multivariable MR analyses adjusted for smoking status & 0.545 \\
Multivariable IVW & $0.948(0.918-0.979)$ & \\
Heterogeneity test statistics & N/A & $\mathbf{0 . 0 0 1}$ \\
Multivariable Egger & $0.936(0.866-1.012)$ & 0.498 \\
Multivariable Egger (intercept) & N/A & 0.095 \\
\hline
\end{tabular}


Table 6. Power calculation of MR analyses

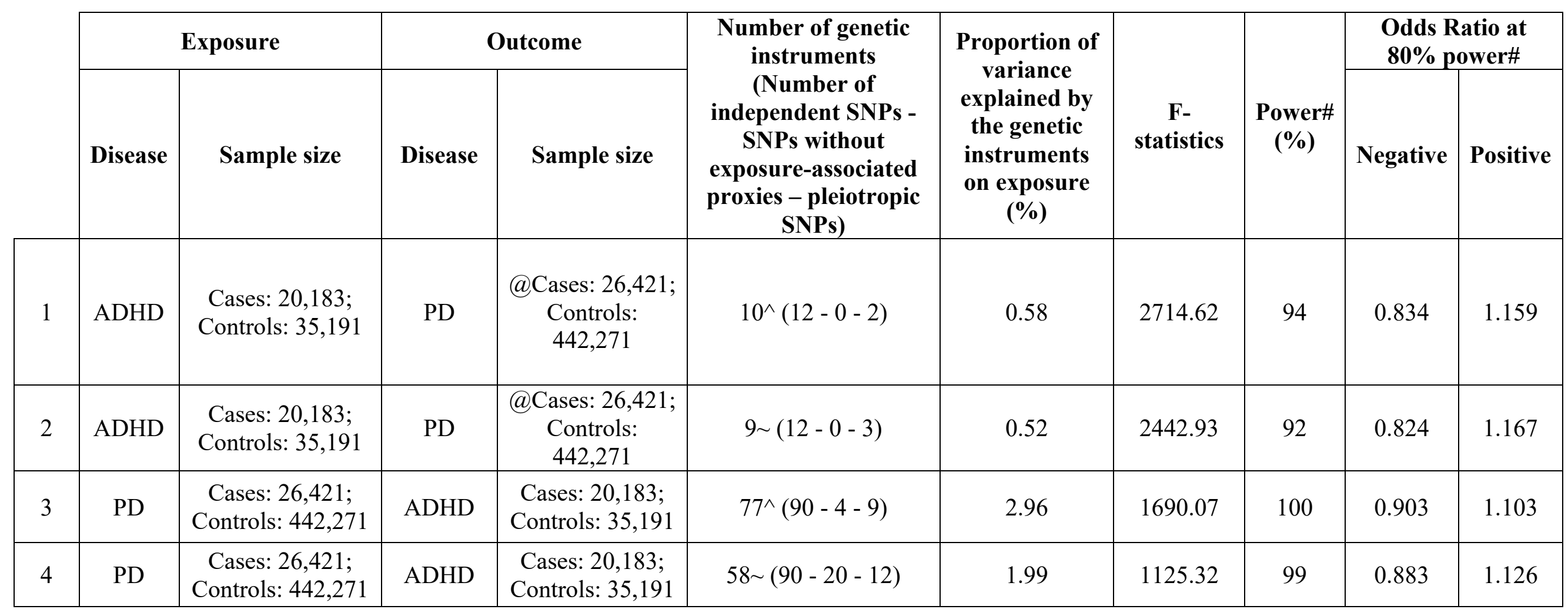

$\wedge$ Genetic instruments were selected by matching across the datasets of ADHD and PD.

$\sim$ Genetic instruments were selected by matching across the datasets of ADHD, PD, BMI and smoking status.

@ Minimum sample size was used to calculate the power

\# Statistical power to detect an odds ratio of 1.2 per doubling of odds of exposure 


\section{Figure Legends}

Figure 1. Key assumptions in MR analyses

(a) Key assumptions of univariable MR analyses and traits included in the current study.

(b) Key assumptions of multivariable MR analyses and traits included in the current study. 


\section{References}

Advokat, C., 2007. Literature Review: Update on Amphetamine Neurotoxicity and Its Relevance to the Treatment of ADHD. Journal of Attention Disorders 11, 8-16. Arnold, L.E., Hodgkins, P., Kahle, J., Madhoo, M., Kewley, G., 2020. Long-Term Outcomes of ADHD: Academic Achievement and Performance. J Atten Disord 24, 73-85.

Arnsten, A.F.T., 2006. Stimulants: Therapeutic Actions in ADHD.

Neuropsychopharmacology : official publication of the American College of Neuropsychopharmacology 31, 2376-2383.

Ascherio, A., Schwarzschild, M.A., 2016. The epidemiology of Parkinson's disease: risk factors and prevention. Lancet Neurol 15, 1257-1272.

Attention-Deficit, S.O., DISORDER, H.J.P., 2011. ADHD: clinical practice guideline for the diagnosis, evaluation, and treatment of attention-deficit/hyperactivity disorder in children and adolescents. 128, 1007.

Banerjee, T.D., Middleton, F., Faraone, S.V., 2007. Environmental risk factors for attentiondeficit hyperactivity disorder. Acta paediatrica (Oslo, Norway : 1992) 96, 1269-1274.

Bonvicini, C., Faraone, S.V., Scassellati, C., 2016. Attention-deficit hyperactivity disorder in adults: A systematic review and meta-analysis of genetic, pharmacogenetic and biochemical studies. Mol Psychiatry 21, 872-884.

Bowden, J., Davey Smith, G., Burgess, S., 2015. Mendelian randomization with invalid instruments: effect estimation and bias detection through Egger regression. Int J Epidemiol 44, 512-525.

Bowden, J., Davey Smith, G., Haycock, P.C., Burgess, S., 2016. Consistent Estimation in Mendelian Randomization with Some Invalid Instruments Using a Weighted Median Estimator. Genet Epidemiol 40, 304-314.

Brion, M.J., Shakhbazov, K., Visscher, P.M., 2013. Calculating statistical power in Mendelian randomization studies. Int J Epidemiol 42, 1497-1501.

Bulik-Sullivan, B., Finucane, H.K., Anttila, V., Gusev, A., Day, F.R., Loh, P.R., ReproGen, C., Psychiatric Genomics, C., Genetic Consortium for Anorexia Nervosa of the Wellcome Trust Case Control, C., Duncan, L., Perry, J.R., Patterson, N., Robinson, E.B., Daly, M.J., Price, A.L., Neale, B.M., 2015a. An atlas of genetic correlations across human diseases and traits. Nat Genet 47, 1236-1241.

Bulik-Sullivan, B.K., Loh, P.R., Finucane, H.K., Ripke, S., Yang, J., Schizophrenia Working Group of the Psychiatric Genomics, C., Patterson, N., Daly, M.J., Price, A.L., Neale, B.M., 2015b. LD Score regression distinguishes confounding from polygenicity in genome-wide association studies. Nat Genet 47, 291-295.

Burgess, S., Butterworth, A., Thompson, S.G., 2013. Mendelian randomization analysis with multiple genetic variants using summarized data. Genet Epidemiol 37, 658-665.

Burgess, S., Davies, N.M., Thompson, S.G., 2016. Bias due to participant overlap in twosample Mendelian randomization. Genet Epidemiol 40, 597-608.

Burgess, S., Foley, C.N., Allara, E., Staley, J.R., Howson, J.M.M., 2020. A robust and efficient method for Mendelian randomization with hundreds of genetic variants. Nat Commun 11, 376.

Burgess, S., Labrecque, J.A., 2018. Mendelian randomization with a binary exposure variable: interpretation and presentation of causal estimates. Eur J Epidemiol 33, 947-952. 
Burgess, S., Thompson, D.J., Rees, J.M.B., Day, F.R., Perry, J.R., Ong, K.K., 2017. Dissecting Causal Pathways Using Mendelian Randomization with Summarized Genetic Data:

Application to Age at Menarche and Risk of Breast Cancer. Genetics 207, 481-487.

Burgess, S., Thompson, S.G., 2015. Multivariable Mendelian randomization: the use of pleiotropic genetic variants to estimate causal effects. Am J Epidemiol 181, 251-260. Chang, C.C., Chow, C.C., Tellier, L.C., Vattikuti, S., Purcell, S.M., Lee, J.J., 2015. Secondgeneration PLINK: rising to the challenge of larger and richer datasets. Gigascience 4, 7. Chen, H., Zhang, S.M., Schwarzschild, M.A., Hernan, M.A., Willett, W.C., Ascherio, A., 2004. Obesity and the risk of Parkinson's disease. Am J Epidemiol 159, 547-555.

Cortese, S., Adamo, N., Del Giovane, C., Mohr-Jensen, C., Hayes, A.J., Carucci, S., Atkinson, L.Z., Tessari, L., Banaschewski, T., Coghill, D., Hollis, C., Simonoff, E., Zuddas, A., Barbui, C., Purgato, M., Steinhausen, H.C., Shokraneh, F., Xia, J., Cipriani, A., 2018. Comparative efficacy and tolerability of medications for attention-deficit hyperactivity disorder in children, adolescents, and adults: a systematic review and network meta-analysis. Lancet Psychiatry 5, 727-738.

Curtin, K., Fleckenstein, A.E., Keeshin, B.R., Yurgelun-Todd, D.A., Renshaw, P.F., Smith, K.R., Hanson, G.R., 2018. Increased risk of diseases of the basal ganglia and cerebellum in patients with a history of attention-deficit/hyperactivity disorder.

Neuropsychopharmacology : official publication of the American College of Neuropsychopharmacology 43, 2548-2555.

Davies, N.M., Holmes, M.V., Davey Smith, G., 2018. Reading Mendelian randomisation studies: a guide, glossary, and checklist for clinicians. BMJ 362, k601.

De Lau, L.M., Breteler, M.M.J.T.L.N., 2006. Epidemiology of Parkinson's disease. 5, 525-535. Demontis, D., Walters, R.K., Martin, J., Mattheisen, M., Als, T.D., Agerbo, E., Baldursson, G., Belliveau, R., Bybjerg-Grauholm, J., Baekvad-Hansen, M., Cerrato, F., Chambert, K., Churchhouse, C., Dumont, A., Eriksson, N., Gandal, M., Goldstein, J.I., Grasby, K.L., Grove, J., Gudmundsson, O.O., Hansen, C.S., Hauberg, M.E., Hollegaard, M.V., Howrigan, D.P., Huang, H., Maller, J.B., Martin, A.R., Martin, N.G., Moran, J., Pallesen, J., Palmer, D.S., Pedersen, C.B., Pedersen, M.G., Poterba, T., Poulsen, J.B., Ripke, S., Robinson, E.B., Satterstrom, F.K., Stefansson, H., Stevens, C., Turley, P., Walters, G.B., Won, H., Wright, M.J., Consortium, A.W.G.o.t.P.G., Early, L., Genetic Epidemiology, C., andMe Research, T., Andreassen, O.A., Asherson, P., Burton, C.L., Boomsma, D.I., Cormand, B., Dalsgaard, S., Franke, B., Gelernter, J., Geschwind, D., Hakonarson, H., Haavik, J., Kranzler, H.R., Kuntsi, J., Langley, K., Lesch, K.P., Middeldorp, C., Reif, A., Rohde, L.A., Roussos, P., Schachar, R., Sklar, P., Sonuga-Barke, E.J.S., Sullivan, P.F., Thapar, A., Tung, J.Y., Waldman, I.D., Medland, S.E., Stefansson, K., Nordentoft, M., Hougaard, D.M., Werge, T., Mors, O., Mortensen, P.B., Daly, M.J., Faraone, S.V., Borglum, A.D., Neale, B.M., 2019. Discovery of the first genome-wide significant risk loci for attention deficit/hyperactivity disorder. Nat Genet 51, 63-75.

Dorsey, E.R., Elbaz, A., Nichols, E., Abd-Allah, F., Abdelalim, A., Adsuar, J.C., Ansha, M.G., Brayne, C., Choi, J.-Y.J., Collado-Mateo, D.J.T.L.N., 2018. Global, regional, and national burden of Parkinson's disease, 1990-2016: a systematic analysis for the global burden of disease study 2016. 17, 939-953.

Emdin, C.A., Khera, A.V., Kathiresan, S., 2017. Mendelian Randomization. JAMA 318, 19251926.

Fuemmeler, B.F., Ostbye, T., Yang, C., McClernon, F.J., Kollins, S.H., 2011. Association between attention-deficit/hyperactivity disorder symptoms and obesity and hypertension in early adulthood: a population-based study. Int J Obes (Lond) 35, 852-862. 
Geissler, J.M., Romanos, M., Gerlach, M., Berg, D., Schulte, C., 2017. No genetic association between attention-deficit/hyperactivity disorder (ADHD) and Parkinson's disease in nine ADHD candidate SNPs. Attention deficit and hyperactivity disorders 9, 121-127.

Gerlach, M., Bonoschewski, T., Warnkel, A., Rothenberger, A., 2003. Parkinson's disease following methylphenidate administration in childhood? An empirical assessment of the current situation.

Gorell, J.M., Johnson, C.C., Rybicki, B.A., Peterson, E.L., Kortsha, G.X., Brown, G.G., Richardson, R.J., 1997. Occupational exposures to metals as risk factors for Parkinson's disease. 48, 650-658.

Gudmundsson, O.O., Walters, G.B., Ingason, A., Johansson, S., Zayats, T., Athanasiu, L., Sonderby, I.E., Gustafsson, O., Nawaz, M.S., Jonsson, G.F., Jonsson, L., Knappskog, P.M., Ingvarsdottir, E., Davidsdottir, K., Djurovic, S., Knudsen, G.P.S., Askeland, R.B., Haraldsdottir, G.S., Baldursson, G., Magnusson, P., Sigurdsson, E., Gudbjartsson, D.F., Stefansson, H., Andreassen, O.A., Haavik, J., Reichborn-Kjennerud, T., Stefansson, K., 2019. Attention-deficit hyperactivity disorder shares copy number variant risk with schizophrenia and autism spectrum disorder. Transl Psychiatry 9, 258.

Hegvik, T.A., Instanes, J.T., Haavik, J., Klungsoyr, K., Engeland, A., 2018. Associations between attention-deficit/hyperactivity disorder and autoimmune diseases are modified by sex: a population-based cross-sectional study. Eur Child Adolesc Psychiatry 27, 663-675. Hemani, G., Zheng, J., Elsworth, B., Wade, K.H., Haberland, V., Baird, D., Laurin, C., Burgess, S., Bowden, J., Langdon, R., Tan, V.Y., Yarmolinsky, J., Shihab, H.A., Timpson, N.J., Evans, D.M., Relton, C., Martin, R.M., Davey Smith, G., Gaunt, T.R., Haycock, P.C., 2018. The MRBase platform supports systematic causal inference across the human phenome. Elife 7. Hubble, J.P., Cao, T., Hassanein, R.E.S., Neuberger, J.S., Roller, W.C., 1993. Risk factors for Parkinson's disease. 43, 1693-1693.

Hui, K.Y., Fernandez-Hernandez, H., Hu, J., Schaffner, A., Pankratz, N., Hsu, N.Y., Chuang, L.S., Carmi, S., Villaverde, N., Li, X., Rivas, M., Levine, A.P., Bao, X., Labrias, P.R., Haritunians, T., Ruane, D., Gettler, K., Chen, E., Li, D., Schiff, E.R., Pontikos, N., Barzilai, N., Brant, S.R., Bressman, S., Cheifetz, A.S., Clark, L.N., Daly, M.J., Desnick, R.J., Duerr, R.H., Katz, S., Lencz, T., Myers, R.H., Ostrer, H., Ozelius, L., Payami, H., Peter, Y., Rioux, J.D., Segal, A.W., Scott, W.K., Silverberg, M.S., Vance, J.M., Ubarretxena-Belandia, I., Foroud, T., Atzmon, G., Pe'er, I., loannou, Y., McGovern, D.P.B., Yue, Z., Schadt, E.E., Cho, J.H., Peter, I., 2018. Functional variants in the LRRK2 gene confer shared effects on risk for Crohn's disease and Parkinson's disease. Sci Transl Med 10.

Jostins, L., Levine, A.P., Barrett, J.C., 2013. Using genetic prediction from known complex disease Loci to guide the design of next-generation sequencing experiments. PLoS One 8 , e76328.

Knouse, L.E., Traeger, L., O'Cleirigh, C., Safren, S.A., 2013. Adult attention deficit hyperactivity disorder symptoms and five-factor model traits in a clinical sample: a structural equation modeling approach. J Nerv Ment Dis 201, 848-854.

Kotagal, V., Bohnen, N.I., Muller, M.L., Koeppe, R.A., Frey, K.A., Langa, K.M., Albin, R.L., 2015. Educational attainment and motor burden in Parkinson's disease. Mov Disord 30, 1143-1147.

Levy, F.J.A., Psychiatry, N.Z.J.o., 1991. The dopamine theory of attention deficit hyperactivity disorder (ADHD). 25, 277-283.

Liu, M., Jiang, Y., Wedow, R., Li, Y., Brazel, D.M., Chen, F., Datta, G., Davila-Velderrain, J., McGuire, D., Tian, C., Zhan, X., andMe Research, T., Psychiatry, H.A.-I., Choquet, H., 
Docherty, A.R., Faul, J.D., Foerster, J.R., Fritsche, L.G., Gabrielsen, M.E., Gordon, S.D., Haessler, J., Hottenga, J.J., Huang, H., Jang, S.K., Jansen, P.R., Ling, Y., Magi, R., Matoba, N., McMahon, G., Mulas, A., Orru, V., Palviainen, T., Pandit, A., Reginsson, G.W., Skogholt, A.H., Smith, J.A., Taylor, A.E., Turman, C., Willemsen, G., Young, H., Young, K.A., Zajac, G.J.M., Zhao, W., Zhou, W., Bjornsdottir, G., Boardman, J.D., Boehnke, M., Boomsma, D.I., Chen, C., Cucca, F., Davies, G.E., Eaton, C.B., Ehringer, M.A., Esko, T., Fiorillo, E., Gillespie, N.A., Gudbjartsson, D.F., Haller, T., Harris, K.M., Heath, A.C., Hewitt, J.K., Hickie, I.B., Hokanson, J.E., Hopfer, C.J., Hunter, D.J., Iacono, W.G., Johnson, E.O., Kamatani, Y., Kardia, S.L.R., Keller, M.C., Kellis, M., Kooperberg, C., Kraft, P., Krauter, K.S., Laakso, M., Lind, P.A., Loukola, A., Lutz, S.M., Madden, P.A.F., Martin, N.G., McGue, M., McQueen, M.B., Medland, S.E., Metspalu, A., Mohlke, K.L., Nielsen, J.B., Okada, Y., Peters, U., Polderman, T.J.C., Posthuma, D., Reiner, A.P., Rice, J.P., Rimm, E., Rose, R.J., Runarsdottir, V., Stallings, M.C., Stancakova, A., Stefansson, H., Thai, K.K., Tindle, H.A., Tyrfingsson, T., Wall, T.L., Weir, D.R., Weisner, C., Whitfield, J.B., Winsvold, B.S., Yin, J., Zuccolo, L., Bierut, L.J., Hveem, K., Lee, J.J., Munafo, M.R., Saccone, N.L., Willer, C.J., Cornelis, M.C., David, S.P., Hinds, D.A., Jorgenson, E., Kaprio, J., Stitzel, J.A., Stefansson, K., Thorgeirsson, T.E., Abecasis, G., Liu, D.J., Vrieze, S., 2019. Association studies of up to 1.2 million individuals yield new insights into the genetic etiology of tobacco and alcohol use. Nat Genet 51, 237-244.

Lopez, P.L., Torrente, F.M., Ciapponi, A., Lischinsky, A.G., Cetkovich-Bakmas, M., Rojas, J.I., Romano, M., Manes, F.F., 2018. Cognitive-behavioural interventions for attention deficit hyperactivity disorder (ADHD) in adults. Cochrane Database Syst Rev 3, CD010840.

Lotharius, J., Brundin, P.J.N.R.N., 2002. Pathogenesis of Parkinson's disease: dopamine, vesicles and $\alpha$-synuclein. 3, 932.

Martins-Silva, T., Vaz, J.D.S., Hutz, M.H., Salatino-Oliveira, A., Genro, J.P., Hartwig, F.P., Moreira-Maia, C.R., Rohde, L.A., Borges, M.C., Tovo-Rodrigues, L., 2019. Assessing causality in the association between attention-deficit/hyperactivity disorder and obesity: a Mendelian randomization study. Int J Obes (Lond).

McClernon, F.J., Kollins, S.H., 2008. ADHD and smoking: from genes to brain to behavior. Ann N Y Acad Sci 1141, 131-147.

Middeldorp, C.M., Hammerschlag, A.R., Ouwens, K.G., Groen-Blokhuis, M.M., Pourcain, B.S., Greven, C.U., Pappa, I., Tiesler, C.M.T., Ang, W., Nolte, I.M., Vilor-Tejedor, N., Bacelis, J., Ebejer, J.L., Zhao, H., Davies, G.E., Ehli, E.A., Evans, D.M., Fedko, I.O., Guxens, M., Hottenga, J.J., Hudziak, J.J., Jugessur, A., Kemp, J.P., Krapohl, E., Martin, N.G., Murcia, M., Myhre, R., Ormel, J., Ring, S.M., Standl, M., Stergiakouli, E., Stoltenberg, C., Thiering, E., Timpson, N.J., Trzaskowski, M., van der Most, P.J., Wang, C., Genetics, E.A., Lifecourse Epidemiology, C., Psychiatric Genomics Consortium, A.W.G., Nyholt, D.R., Medland, S.E., Neale, B., Jacobsson, B., Sunyer, J., Hartman, C.A., Whitehouse, A.J.O., Pennell, C.E., Heinrich, J., Plomin, R., Smith, G.D., Tiemeier, H., Posthuma, D., Boomsma, D.I., 2016. A Genome-Wide Association MetaAnalysis of Attention-Deficit/Hyperactivity Disorder Symptoms in Population-Based Pediatric Cohorts. J Am Acad Child Adolesc Psychiatry 55, 896-905 e896.

Mok, K.Y., Sheerin, U., Simon-Sanchez, J., Salaka, A., Chester, L., Escott-Price, V., Mantripragada, K., Doherty, K.M., Noyce, A.J., Mencacci, N.E., Lubbe, S.J., International Parkinson's Disease Genomics, C., Williams-Gray, C.H., Barker, R.A., van Dijk, K.D., Berendse, H.W., Heutink, P., Corvol, J.C., Cormier, F., Lesage, S., Brice, A., Brockmann, K., Schulte, C., Gasser, T., Foltynie, T., Limousin, P., Morrison, K.E., Clarke, C.E., Sawcer, S., Warner, T.T., Lees, A.J., Morris, H.R., Nalls, M.A., Singleton, A.B., Hardy, J., Abramov, A.Y., Plagnol, V., 
Williams, N.M., Wood, N.W., 2016. Deletions at 22q11.2 in idiopathic Parkinson's disease: a combined analysis of genome-wide association data. Lancet Neurol 15, 585-596.

Nalls, M.A., Blauwendraat, C., Vallerga, C.L., Heilbron, K., Bandres-Ciga, S., Chang, D., Tan, M., Kia, D.A., Noyce, A.J., Xue, A., Bras, J., Young, E., von Coelln, R., Simon-Sanchez, J., Schulte, C., Sharma, M., Krohn, L., Pihlstrom, L., Siitonen, A., Iwaki, H., Leonard, H., Faghri, F., Gibbs, J.R., Hernandez, D.G., Scholz, S.W., Botia, J.A., Martinez, M., Corvol, J.C., Lesage, S., Jankovic, J., Shulman, L.M., Sutherland, M., Tienari, P., Majamaa, K., Toft, M., Andreassen, O.A., Bangale, T., Brice, A., Yang, J., Gan-Or, Z., Gasser, T., Heutink, P., Shulman, J.M., Wood, N.W., Hinds, D.A., Hardy, J.A., Morris, H.R., Gratten, J., Visscher, P.M., Graham, R.R., Singleton, A.B., andMe Research, T., System Genomics of Parkinson's Disease, C., International Parkinson's Disease Genomics, C., 2019. Identification of novel risk loci, causal insights, and heritable risk for Parkinson's disease: a meta-analysis of genome-wide association studies. Lancet Neurol 18, 1091-1102.

Nalls, M.A., Pankratz, N., Lill, C.M., Do, C.B., Hernandez, D.G., Saad, M., DeStefano, A.L., Kara, E., Bras, J., Sharma, M., Schulte, C., Keller, M.F., Arepalli, S., Letson, C., Edsall, C., Stefansson, H., Liu, X., Pliner, H., Lee, J.H., Cheng, R., International Parkinson's Disease Genomics, C., Parkinson's Study Group Parkinson's Research: The Organized, G.I., andMe, GenePd, NeuroGenetics Research, C., Hussman Institute of Human, G., Ashkenazi Jewish Dataset, I., Cohorts for, H., Aging Research in Genetic, E., North American Brain Expression, C., United Kingdom Brain Expression, C., Greek Parkinson's Disease, C., Alzheimer Genetic Analysis, G., Ikram, M.A., Ioannidis, J.P., Hadjigeorgiou, G.M., Bis, J.C., Martinez, M., Perlmutter, J.S., Goate, A., Marder, K., Fiske, B., Sutherland, M., Xiromerisiou, G., Myers, R.H., Clark, L.N., Stefansson, K., Hardy, J.A., Heutink, P., Chen, H., Wood, N.W., Houlden, H., Payami, H., Brice, A., Scott, W.K., Gasser, T., Bertram, L., Eriksson, N., Foroud, T., Singleton, A.B., 2014. Large-scale meta-analysis of genome-wide association data identifies six new risk loci for Parkinson's disease. Nat Genet 46, 989-993.

Noyce, A.J., Kia, D.A., Hemani, G., Nicolas, A., Price, T.R., De Pablo-Fernandez, E., Haycock, P.C., Lewis, P.A., Foltynie, T., Davey Smith, G., International Parkinson Disease Genomics, C., Schrag, A., Lees, A.J., Hardy, J., Singleton, A., Nalls, M.A., Pearce, N., Lawlor, D.A., Wood, N.W., 2017. Estimating the causal influence of body mass index on risk of Parkinson disease: A Mendelian randomisation study. PLoS Med 14, e1002314.

Polanczyk, G.V., Willcutt, E.G., Salum, G.A., Kieling, C., Rohde, L.A., 2014. ADHD prevalence estimates across three decades: an updated systematic review and meta-regression analysis. Int J Epidemiol 43, 434-442.

Priyadarshi, A., Khuder, S.A., Schaub, E.A., Priyadarshi, S.S., 2001. Environmental Risk Factors and Parkinson's Disease: A Metaanalysis. Environmental Research 86, 122-127. Raman, S.R., Man, K.K.C., Bahmanyar, S., Berard, A., Bilder, S., Boukhris, T., Bushnell, G., Crystal, S., Furu, K., KaoYang, Y.H., Karlstad, O., Kieler, H., Kubota, K., Lai, E.C., Martikainen, J.E., Maura, G., Moore, N., Montero, D., Nakamura, H., Neumann, A., Pate, V., Pottegard, A., Pratt, N.L., Roughead, E.E., Macias Saint-Gerons, D., Sturmer, T., Su, C.C., Zoega, H., Sturkenbroom, M., Chan, E.W., Coghill, D., Ip, P., Wong, I.C.K., 2018. Trends in attentiondeficit hyperactivity disorder medication use: a retrospective observational study using population-based databases. Lancet Psychiatry 5, 824-835.

Rees, J.M.B., Wood, A.M., Burgess, S., 2017. Extending the MR-Egger method for multivariable Mendelian randomization to correct for both measured and unmeasured pleiotropy. Stat Med 36, 4705-4718. 
Sieurin, J., Gustavsson, P., Weibull, C.E., Feldman, A.L., Petzinger, G.M., Gatz, M., Pedersen, N.L., Wirdefeldt, K., 2016. Personality traits and the risk for Parkinson disease: a prospective study. Eur J Epidemiol 31, 169-175.

Simon-Sanchez, J., Schulte, C., Bras, J.M., Sharma, M., Gibbs, J.R., Berg, D., Paisan-Ruiz, C., Lichtner, P., Scholz, S.W., Hernandez, D.G., Kruger, R., Federoff, M., Klein, C., Goate, A., Perlmutter, J., Bonin, M., Nalls, M.A., Illig, T., Gieger, C., Houlden, H., Steffens, M., Okun, M.S., Racette, B.A., Cookson, M.R., Foote, K.D., Fernandez, H.H., Traynor, B.J., Schreiber, S., Arepalli, S., Zonozi, R., Gwinn, K., van der Brug, M., Lopez, G., Chanock, S.J., Schatzkin, A., Park, Y., Hollenbeck, A., Gao, J., Huang, X., Wood, N.W., Lorenz, D., Deuschl, G., Chen, H., Riess, O., Hardy, J.A., Singleton, A.B., Gasser, T., 2009. Genome-wide association study reveals genetic risk underlying Parkinson's disease. Nat Genet 41, 1308-1312.

Staley, J.R., Blackshaw, J., Kamat, M.A., Ellis, S., Surendran, P., Sun, B.B., Paul, D.S., Freitag, D., Burgess, S., Danesh, J., Young, R., Butterworth, A.S., 2016. PhenoScanner: a database of human genotype-phenotype associations. Bioinformatics 32, 3207-3209.

Thomas, R., Sanders, S., Doust, J., Beller, E., Glasziou, P., 2015. Prevalence of attentiondeficit/hyperactivity disorder: a systematic review and meta-analysis. Pediatrics 135, e9941001.

Tripp, G., Wickens, J.R., 2009. Neurobiology of ADHD. Neuropharmacology 57, 579-589. Ugur, C., Uneri, O.S., Goker, Z., Sekmen, E., Aydemir, H., Solmaz, E., 2018. The assessment of serum lipid profiles of children with attention deficit hyperactivity disorder. Psychiatry Res 264, 231-235.

Verbanck, M., Chen, C.Y., Neale, B., Do, R., 2018. Detection of widespread horizontal pleiotropy in causal relationships inferred from Mendelian randomization between complex traits and diseases. Nat Genet 50, 693-698.

Villumsen, M., Aznar, S., Pakkenberg, B., Jess, T., Brudek, T., 2019. Inflammatory bowel disease increases the risk of Parkinson's disease: a Danish nationwide cohort study 19772014. Gut 68, 18-24.

Volkow, N.D., Wang, G.-J., Fowler, J.S., Ding, Y.-S.J.B.p., 2005. Imaging the effects of methylphenidate on brain dopamine: new model on its therapeutic actions for attentiondeficit/hyperactivity disorder. 57, 1410-1415.

Walitza, S., Melfsen, S., Herhaus, G., Scheuerpflug, P., Warnke, A., Muller, T., Lange, K.W., Gerlach, M., 2007. Association of Parkinson's disease with symptoms of attention deficit hyperactivity disorder in childhood. Journal of neural transmission. Supplementum, 311315.

Wang, G.J., Volkow, N.D., Wigal, T., Kollins, S.H., Newcorn, J.H., Telang, F., Logan, J., Jayne, M., Wong, C.T., Han, H., Fowler, J.S., Zhu, W., Swanson, J.M., 2013. Long-term stimulant treatment affects brain dopamine transporter level in patients with attention deficit hyperactive disorder. PLoS One 8, e63023.

Wong, I.C.K., Banaschewski, T., Buitelaar, J., Cortese, S., Dopfner, M., Simonoff, E., Coghill, D., European, A.G.G., 2019. Emerging challenges in pharmacotherapy research on attentiondeficit hyperactivity disorder-outcome measures beyond symptom control and clinical trials. Lancet Psychiatry 6, 528-537.

Xicoy, H., Wieringa, B., Martens, G.J.M., 2019. The Role of Lipids in Parkinson's Disease. Cells 8.

Yang, J., Ferreira, T., Morris, A.P., Medland, S.E., Genetic Investigation of, A.T.C., Replication, D.I.G., Meta-analysis, C., Madden, P.A., Heath, A.C., Martin, N.G., Montgomery, G.W.,

Weedon, M.N., Loos, R.J., Frayling, T.M., McCarthy, M.I., Hirschhorn, J.N., Goddard, M.E., 
Visscher, P.M., 2012. Conditional and joint multiple-SNP analysis of GWAS summary statistics identifies additional variants influencing complex traits. Nat Genet 44, 369-375, S361-363.

Yavorska, O.O., Burgess, S., 2017. MendelianRandomization: an R package for performing Mendelian randomization analyses using summarized data. Int J Epidemiol 46, 1734-1739. Yengo, L., Sidorenko, J., Kemper, K.E., Zheng, Z., Wood, A.R., Weedon, M.N., Frayling, T.M., Hirschhorn, J., Yang, J., Visscher, P.M., Consortium, G., 2018. Meta-analysis of genome-wide association studies for height and body mass index in approximately 700000 individuals of European ancestry. Hum Mol Genet 27, 3641-3649.

Yuan, J., McCann, U., Ricaurte, G., 1997. Methylphenidate and brain dopamine neurotoxicity. Brain research 767, 172-175. 